\title{
Rehabilitation of maxillary arch by immediate implant placement and immediate loading using all-on-6 protocol : A case report.
}

\begin{abstract}
:
Edentulism is a current and relevant oral-health problem which has a negative social and psychological effect on patient's quality of life. Implant-supported fixed restoration is a well-established treatment method for edentulous patients. Current concepts and techniques in implant dentistry have developed and changed with time to provide better esthetics and functional outcomes quickly. Immediate loading offers several advantages over conventional loading without compromising the results. An immediate provisional fixed prosthesis promotes a high level of patient satisfaction with respect to esthetics, phonetics, mastication and psychological comfort, enabling patients to return to their normal routine faster. Other advantages to extraction with simultaneous replacement includes the maintenance of vertical dimension, elimination of interim denture therapy, and potential improvement of soft tissue healing.

This case report demonstrates the treatment modality of immediate implant supported prosthesis based on all-on- 6 protocol. The patient demanded a possible treatment that would replace her dentition on the same day when she leaves the clinic solving the problem of mastication and aesthetics.
\end{abstract}

Keywords: All-on-6 immediate implant, Immediate loading, Immediate functional restoration.

\section{Introduction:}

Oral health is one of the most important factor for general health status, as well as quality of life. Partial or complete edentulism is prevalent worldwide particularly in the 60 years and older age group.[1] It is mainly attributed to patient's neglected behaviour towards decayed teeth, failing restorations and gum diseases. Age, gender, low family income and rural domicile have also been associated with edentulism.[2]

Different rehabilitative options may be chosen to treat complete or partial edentulism such as a complete removable denture, an implant-retained removable denture, and an implant-supported fixed prosthesis.[3] Implants has revolutionised the field of dentistry by showing reliable and predictable results for both partially and fully edentulous patients.[4] The earlier concepts of restoration over implants suggested a loading protocol after 3-6 months or to extract decayed teeth followed by healing of sockets and then implant

\begin{tabular}{|l|c|}
\hline \multicolumn{3}{|c|}{ Access this article online } \\
\hline \multirow{2}{*}{$\begin{array}{l}\text { Website: } \\
\text { www.ujds.in }\end{array}$} & Quick Response Code \\
\hline $\begin{array}{l}\text { DOI: } \\
\text { https://doi.org/10.21276/10.21276/ujds.2020.6.2.11 }\end{array}$ & \\
\hline
\end{tabular}

placement.( Table 1)[5] Ever since clinicians are trying the possibilities to shorten the treatment time for implants by placing them in fresh extraction sockets and immediate restoration or loading. (Table 2[6], Box 1)

This article presents case report on immediate implant placement in fresh extraction sockets followed by immediate loading using splinted acrylic framework over all-on-6

\section{${ }^{1}$ SHARMA A, ${ }^{2}$ SETHI N, ${ }^{3}$ GUPTA R, ${ }^{4}$ SINGH JABBAL, $R$ \\ 1,2,3 Department of Prosthodontics and Implantology, Himachal Institute of Dental Sciences, Paonta Sahib ${ }^{4}$ Department of Prosthodontics and Implantology, Genesis Institute of Dental Sciences and Research, Moga Road, Ferozepur}

Address for Correspondence: Dr. Aastha Sharma Department of Prosthodontics and Implantology, Himachal Institute of Dental Sciences, Paonta Sahib, Himachal Pradesh

Email: aasthasharma1805@gmail.com, nits_1011@yahoo.co.in

Received : 8 July 2020, Published : 31 August 2020

How to cite this article: Sharma, A., Sethi, N., Gupta, R., \& Singh Jabbal, R. (2020). Rehabilitation of maxillary arch by immediate implant placement and immediate loading using all on 6 protocol : A case report. UNIVERSITY JOURNAL OF DENTAL SCIENCES, 6(2):63-7. 
implants using multiangle abutments within 24 hours. Final outcome was successful integration of implants after 3 months.

\begin{tabular}{|l|l|l|}
\hline $\begin{array}{l}\text { Table 1 - Terms related to loading protocols } \\
\text { recommended by Cochran et al }\end{array}$ & $\begin{array}{l}\text { Provisional } \\
\text { restoration acclusion with } \\
\text { delivered after } \\
\text { implant placement }\end{array}$ & $\begin{array}{l}\text { Opposite arch } \\
\text { oplat }\end{array}$ \\
\hline $\begin{array}{l}\text { Immediate restoration/ } \\
\text { immediate provisionalization }\end{array}$ & Within $48 \mathrm{hrs}$ & Absent \\
\hline Immediate loading & Within 48 hrs & Present \\
\hline Early loading & 48 hrs to 3 months & Present \\
\hline Conventional loading & $3-6$ months & present \\
\hline Delayed loading & $>6$ months & present \\
\hline
\end{tabular}

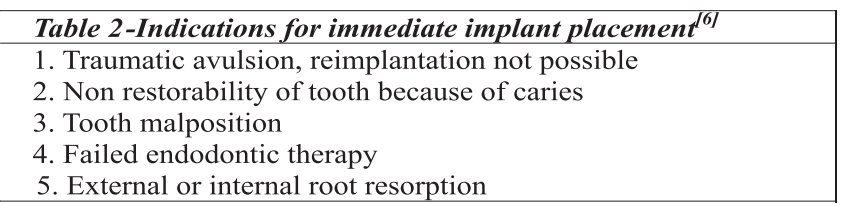

\begin{tabular}{|l|}
\hline Box 1-Advantages of immediate loading \\
- Reduction in alveolar ridge resorption \\
- Reduced surgical trauma and ease of surgery \\
- Avoidance of a removable prosthesis that may \\
interfere with healing \\
- Quicker return of function \\
- solution with good soft tissue profile \\
Psychological benefit resulting in increased patient \\
acceptance \\
Reduction in overall treatment time
\end{tabular}

\section{Case Report:}

A 42 years old female approached outpatient department of Prosthodontics and Implantology at Himachal institute of dental sciences, Paonta sahib, having a complaint of multiple decayed teeth and as an effect had unpleasant aesthetics and difficulty in speaking and eating.. Intraoral examination revealed few remaining natural teeth and grossly decayed teeth in maxillary arch, periodontically compromised lower central incisors and bilaterally missing lower posteriors $(36,45,46)$. (Fig $1 \mathrm{a}, \mathrm{b})$ The patient had a history of mild hypertension and uncontrolled blood glucose level. The patient requested a fixed rehabilitaion without being noticed as edentulous. Then the treatment option of immediate extraction and immediate loading over 6 implants in maxillary arch was explained to the patient, followed by replacement of missing \doomed for extraction mandibular teeth. Tooth no. 28 was intentionally saved as it would help her in chewing during recovery phase and maintain vertical dimension. The remaining maxillary teeth $(11,21,23)$ appeared not to be suitable for fixed treatment because of history of repeated sinus formation and their position, they wouldn't allow for placement of single piece prosthesis and cross arch stabalisation, therefore, it was decided to remove them. After patient's consent, initial treatment started with diagnostics, photographs, radiographs (Panaromic and full mouth Rvgs) and blood glucose level monitoring. (Fig 2)

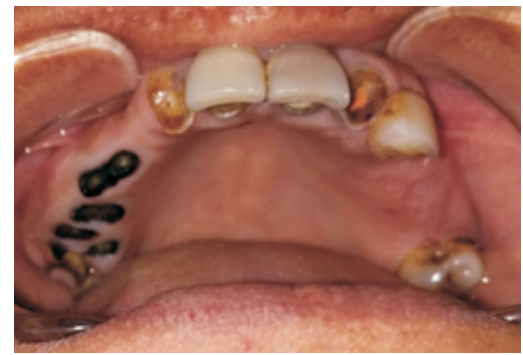

Fig 1 a) Preoperative maxillary arch

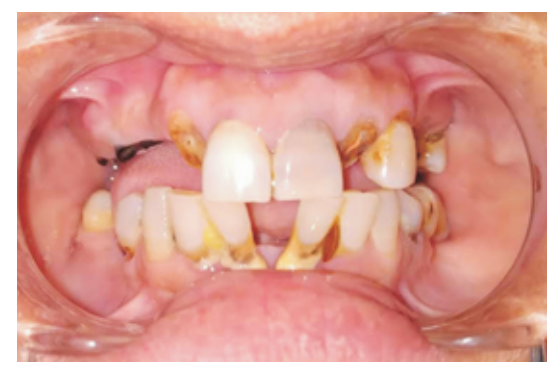

b) Preoperative view in occlusion

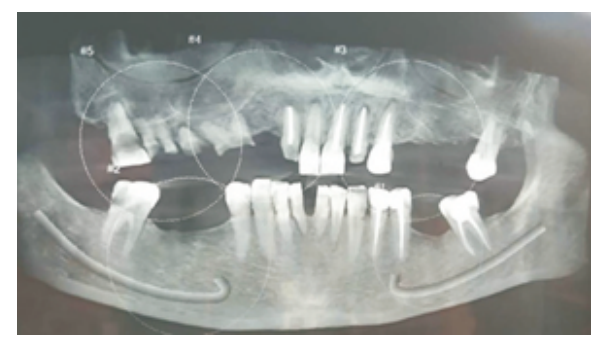

Fig 2. Preoperative panaromic view

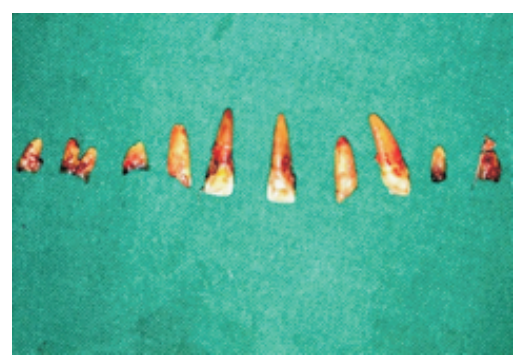

Fig 3. Extracted teeth

After controlling patient's systemic parameters, prophylactic antibiotic coverage ( amoxicillin $1 \mathrm{gms}$ ) was given $2 \mathrm{hrs}$ prior to surgery followed by rinse with chlorhexidine mouth wash $(0.20 \%)$ for 1 minute prior to surgery. Local anaesthesia was administered containing 2\% lignocaine. All the remaining and decayed maxillary teeth were extracted atraumatically, granulation tissue was removed and sockets were cleaned (Fig 3). This was followed by sequential drilling and four implants were placed in axial direction at site 12,11,21,22. (Osteem TS III, size $4 \times 13 \mathrm{~mm}$ ). Two posterior tilted implants were placed at 2 nd premolar areas avoiding sinus lining. After placing implants in sockets and confirming primary torque of 
$35 \mathrm{Ncm}$, multiangle abutments were tightened at $25 \mathrm{Ncm}$ torque and guiding pins were placed to check and maintain relative parallelism of abutments.(Fig 4) In this case a prefabricated polymethyl methacrylate acrylised interim prosthesis was already made with highly polished intaglio surface using diagnostic model and was sterilised by keeping in idophore solution $24 \mathrm{hrs}$ prior to surgery. After confirming parallelism, guiding screws were removed and suturing was done using 4-0 vicryl resorbable sutures to approximate the flaps (Fig 5). Then a thin layered alginate impression was made using prefabricated prosthesis to locate the position of multiunit abutments. The prosthesis was drilled at same sites to make room for temporary prosthetic titanium cylinders. The height of titanium cylinders were adjusted after assessing interarch space (Fig 6,7). Pour type chairside resin was used to attach prosthesis/superstructure with cylinders. Excess material was trimmed and polished and the prosthesis was again seated, screws for seated titanium cylinders tightened with a final torque for 10 seconds each, access holes were blocked and filled with flowable composite resin. Occlusal adjustment was done to get maximum contacts in centric occlusion and canine guided disocclusion. A thorough post operative instruction session was given which includes consumption of soft diet for 3 to 4 weeks, maintenance of oral hygiene using chlorhexidine mouthwash twice a day for 2 weeks and regular followups (Fig 8).

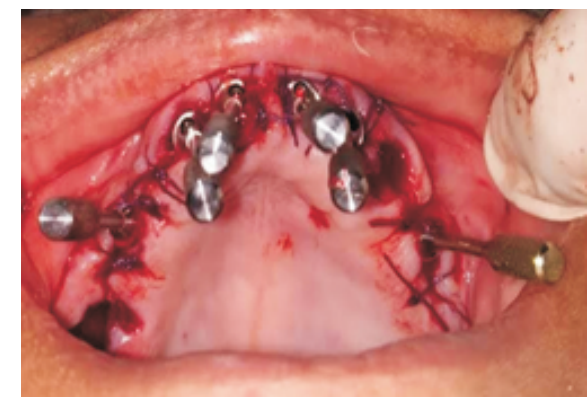

Fig4. Guiding screws over multiunit

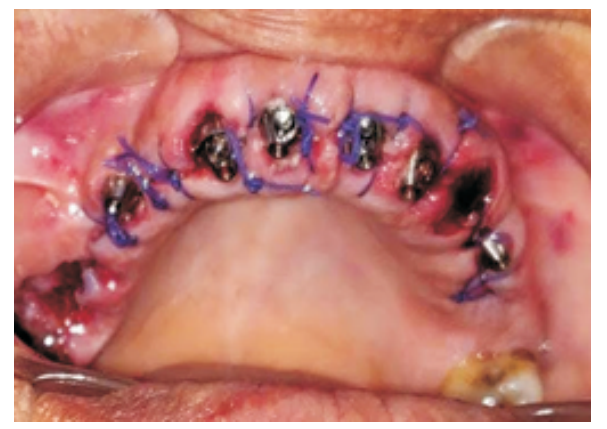

Fig5. Suturing done to approximate wound abutments to maintain parallelism

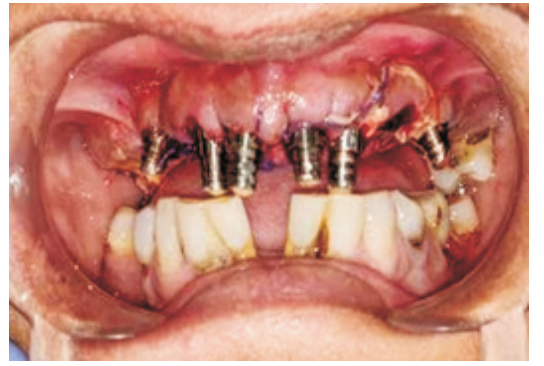

Fig 6 Titanium cylinders placed

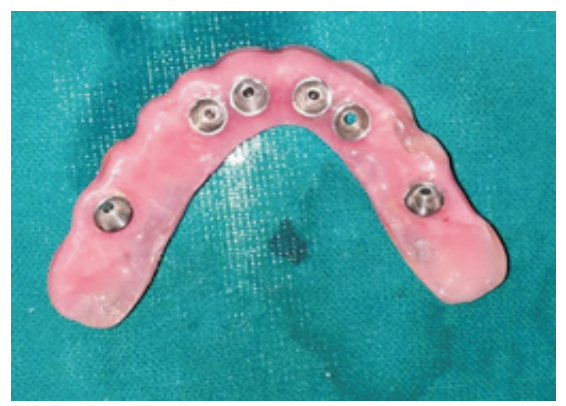

Fig 7 Intaglio surface of prosthesis showing titanium cylinders attached to it.

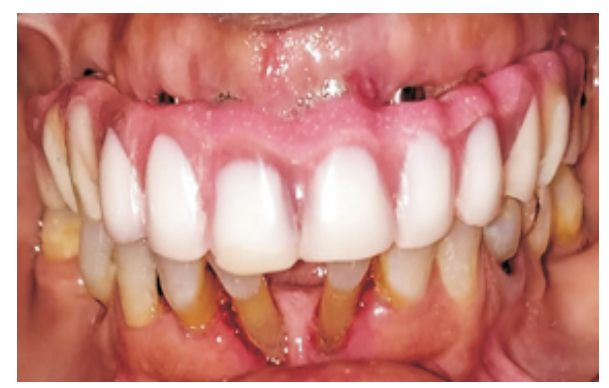

Fig 8. Intrerim prosthesis in occlusion.

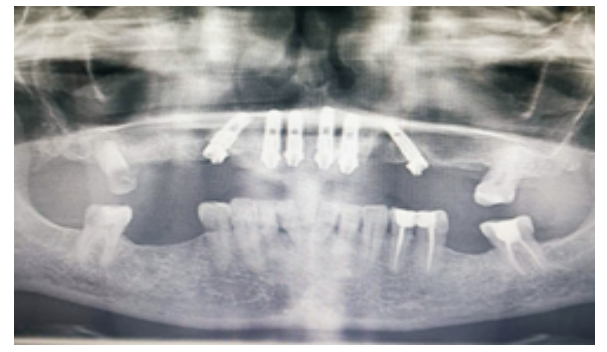

Fig 9. Post operative radiograph

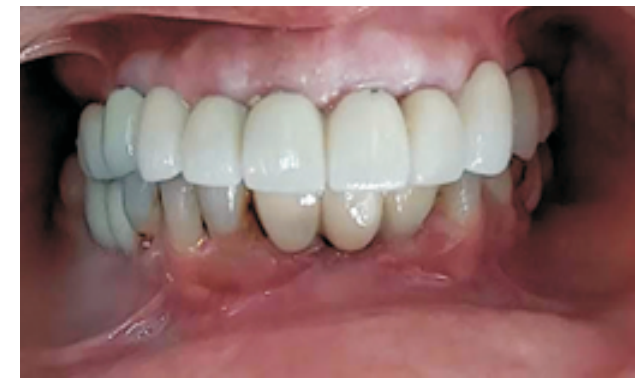

Fig11. Final PFM prosthesis in occlusion 


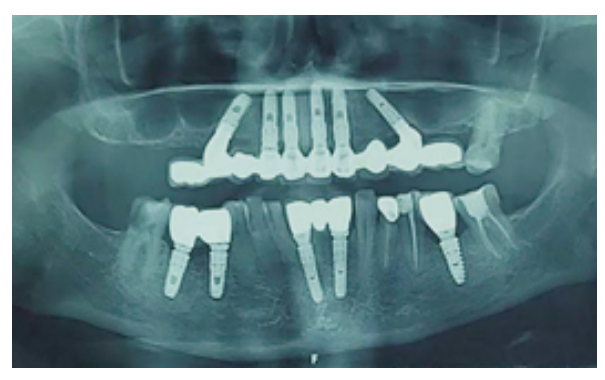

Fig 12-Radiograph after 12 months

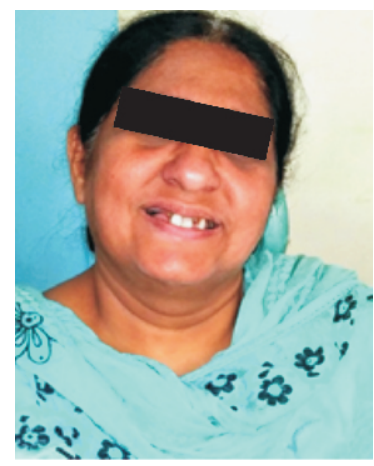

Fig 13 a) Pretreatment extraoral view

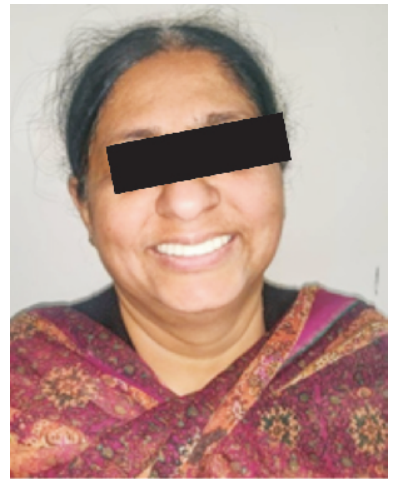

b) After treatment extraoral view

\section{Outcome and Follow-up:}

Follow ups were done after 72hrs, (Fig 9) one week, one month and a final splinted PFM prosthesis was delivered 3 months after implant placement. Lower arch was restored with immediate implant placement w.r.t 32, 41 and flapless implant placement w.r.t 36, 46 and 47. Final lower prosthesis was delivered after 3 months of follow up. (Fig 10-13). After complete rehabilitation the patient was recalled at 6 and 12 months and thereafter, yearly.

\section{Disscussion:}

Rehabilitation of posterior maxilla is often challenging due to resorption of alveolar ridge and sinus pneumatisation.[7] It often presents with poor bone quality and quantity.[8] Treatment options available includes sinus lifting, ridge augmentation, bone grafting, short or zygomatic implants. Tilted implants can be used as an alternative to avoid surgical complexities such as morbidity at the graft donor sites, post operative discomfort, questionable predictability[9], increased costs and prolonged treatment time.[10]

In 2003, Malo and collegues introduced all-on-4 or 6 immediate function concepts. These procedures involve placing 2 or 4 straight implants in the anterior region and 2 implants, tilted at $35^{\circ}$ to $45^{\circ}$, in the posterior region.[11]

There are various biomechanical advantages of posterior tilting of implants as compared to upright position. The distalisation of implant platform reduces the moments of force and improves the load distribution,[12] although it is relatively difficult to insert posterior tilted fixtures. Posterior tilted implant supported prosthesis simply increases anterioposterior spread, therefore shortening the cantilever, increasing cross arch stabilisation and load distribution.[13] All on six is a more viable option from biomechanical point of view as it appears to induce lower stress as compared to all on four concept.[14] A study by S. Wentaschek et al15 showed that immediate splinted loading on 6 implants with tilted distal implants is a predictable treatment modality for edentulous maxilla even if extraction of the remaining teeth and simultaneous implant placement is performed or if very limited bone is available in atrophic jaws. Immediate implant allows preservation of associated hard and soft tissues and minimizes ridge resorption.[16] Although this treatment is technique sensitive but it is less time consuming, less invasive, cost effective and more comfortable in restoring quality of life.[15]

Many systematic and meta analyses [17-19] showed that immediate implant loading does not impair treatment success. Their results pointed on proper patient selection, treatment planning and implant micro morphology for better outcomes. Immediate loading should be done within $48 \mathrm{hrs}$ of implant placement with restoration in occlusion with opposite arch.[20] In case of full arch immediate loading implants should have adequate primary stability at the time of placement, rigid interimplant splinting and occlusal forces should be appropriately controlled during osseointegration period to prevent micromovements. $[10,21]$

\section{Conclusion :}

Correct diagnosis and treatment planning are the key for successful immediate implant rehabilitation in resorbed ridges. The "All-on-6" treatment concept seems to be a viable 
option for rehabilitating edentulous maxillary jaws. However, this approach is considered highly technique sensitive and requires careful selection of cases, proper treatment plan, skilled clinician with more experience and a better understanding of the range of prosthetic components.

\section{References:}

1. Felton David. Edentulism and commorbid factors. J Prosthodontics 2009;18:88-96.

2. Medina-Solís CE, Pérez-Núñez R, Maupomé G et al. Edentulism among Mexican adults aged 35 years and older and associated factors. Am J Public Health 2006;96(9):1578-81.

3. D. A. Felton, "Edentulism and comorbid factors," Journal of Prosthodontics. 2009; vol. 18(2): 88-96.

4. Sean Chung, Anthony McCullagh, Tassos Irinakis. Immediate Loading in the Maxillary Arch: Evidence-Based Guidelines to Improve Success Rates: A Review. Journal of Oral Implantology. 2011; Vol. XXXVII(No. Five):610-21.

5. Cochran DL, Morton D, Weber HP. Consensus statements and recommended clinical procedures regarding loading protocols for endosseous dental implants. Int $\mathrm{J}$ Oral Maxillofac Implants. 2004;19(suppl):109-113.

6. James Parelli, Shelly Abramowicz. Immediate Placement and Immediate Loading Surgical Technique and Clinical Pearls. Dent Clin NAm 59 (2015) 345-355

7. M. Chiapasco, M. Zaniboni, J. Oral Maxillofac. Surg. 67 (2009) 867-871.

8. U. Lekholm, G. Zarb, Patient selection and preparation, in: P.I. Branemark, G.A. Zarb, T. Albrektsson (Eds.), Tissue-integrated Prostheses: Osseointegration in Clinical Dentistry, Quintessence, Chicago 1985, pp. 199-209.

9. Al-Nawas B, Wegener J, Bender C, Wagner W. Critical soft tissue parameters of the zygomatic implant. J Clin Periodontol. 2004;31:497-500

10. Agliardi E, Panigatti S, Clerico ' M et al. Immediate rehabilitation of the edentulous jaws with full fixed prostheses supported by four implants: interim results of a single cohort prospective study. Clin Oral Implants Res. 2010;21:459-465.

11. Malo' P, Nobre MD, Lopes A. Immediate loading of 'all-on-40 maxillary prostheses using trans-sinus tilted implants without sinus bone grafting: a retrospective study reporting the 3-year outcome. Eur J Oral Implantol. 2013;6:273-283

12. Bellini CM, Romeo D, Galbusera F, et al. A finite element analysis of tilted versus nontilted implant configurations in the edentulous maxilla. Int J Prosthodont 2009; 22:155-157.. 20101..10

13. Michael H. Chan, Curtis Holmes. Contemporary "All-on-4" Concept. Dent Clin NAm 59 (2015) 421-470

14. E.O. Almeida, E.P. Rocha, A.C. Freitas Junior, R.B. Anchieta, R. Poveda, N. Gupta, P.G. Coelho, Clin. Implant. Dent. Relat. Res. 17 (Suppl. 1) (2015) e332-e342.

15. S Wentaschek, S Hartmann, C Walter et al. Six-implant-supported immediate fixed rehabilitation of atrophic edentulous maxillae with tilted distal implants International Journal of Implant Dentistry (2017) $3: 35$.

16. Botticelli D, Berglundh T, Lindhe J. Hard-tissue alterations following immediate implant placement in extraction sites. J Clin Periodontol 2004;31:820-8.

17. Ioannidou E, Doufexi A. Does loading time affect implant survival? A meta-analysis of 1,266 implants. J Periodontol 2005; 76:1252-1258.
18. Del Fabbro M, Testori T, Francetti L,et al. Systematic review of survival rates for immediately loaded dental implants. Int J Periodontics Restorative Dent 2006; 26:249-263.

19. Esposito M, Grusovin MG, Achille $\mathrm{H}$,et al. Interventions for replacing missing teeth: different times for loading dental implants. Cochrane Database Syst Rev 2009; :CD003878.

20. Corbella S, Del Fabbro M, Taschieri S et al. Clinical evaluation of an implant maintenance protocol for the prevention of periimplant diseases in patients treated with immediately loaded full-arch rehabilitations. Int J Dent Hyg. 2011;9:216-222.

21. Degidi M, Nardi D, Piattelli A. Immediate loading of the edentulous maxilla with a definitive restoration supported by an intraorally welded titanium bar and tilted implants. Int J Oral Maxillofac Implants. 2010;25:1175-1182. 